\title{
SUPERDIMENSIONAL METAMATERIAL RESONATORS FROM SUB-RIEMANNIAN GEOMETRY
}

\section{Greenleaf, Allan}

2018

Greenleaf , A , Kettunen , H , Kurylev , Y , Lassas , M \& Uhlmann , G 2018 , ' SUPERDIMENSIONAL METAMATERIAL RESONATORS FROM SUB-RIEMANNIAN

GEOMETRY' , SIAM Journal on Applied Mathematics , vol. 78 , no. 1 , pp. 437-456 . https://doi.org/10.1137/17M11

http://hdl.handle.net/10138/326771

https://doi.org/10.1137/17M1130964

unspecified

acceptedVersion

Downloaded from Helda, University of Helsinki institutional repository.

This is an electronic reprint of the original article.

This reprint may differ from the original in pagination and typographic detail.

Please cite the original version. 


\title{
Superdimensional Metamaterial Resonators
}

Allan Greenleaf*, Henrik Kettunen, Yaroslav Kurylev, Matti Lassas and Gunther Uhlmann Department of Mathematics, University of Rochester, Rochester, NY 14627

Department of Mathematics, University of Helsinki, FIN-00014, Finland, Department of Mathematical Sciences, University College London, Gower Str, London, WC1E 6BT, UK

Department of Mathematics, University of Helsinki, FIN-00014, Finland, and Department of Mathematics, University of Washington, Seattle, WA 98195

*Authors are listed in alphabetical order

\begin{abstract}
We propose a fundamentally new method for the design of metamaterial arrays, valid for any waves modeled by the Helmholtz equation, including scalar optics and acoustics. The design and analysis of these devices is based on eigenvalue and eigenfunction asymptotics of solutions to Schrödinger wave equations with harmonic and degenerate potentials. These resonators behave superdimensionally, with a higher local density of eigenvalues and greater concentration of waves than expected from the physical dimension, e.g., planar resonators function as 3- or higher-dimensional media, and bulk material as effectively of dimension 4 or higher. Applications include antennas with a high density of resonant frequencies and giant focussing, and are potentially broadband.
\end{abstract}

PACS numbers: 84.40.Ba, 78.67.Pt, 42.79.Ry 
1. Introduction. The advent of transformation optics has resulted in numerous theoretical designs allowing extreme manipulation of waves, including cloaks [1 3], field rotators [4], electromagnetic wormholes [5] and illusion optics [6], among many others. The ongoing development of metamaterials has allowed some of these plans to be implemented in at least a reasonable approximation to the theoretically perfect ideal [7, 8]. Since a partial differential equation may describe a variety of physical waves, one theoretical transformation optics design may in principle be implemented for a number of distinct wave phenomena. Thus, a Helmholtz equation design can be applied to scalar optics [2], electromagnetism in cylindrical geometry, acoustics [9], small amplitude water waves [10], and, via homogenization and a gauge transformation, even matter waves in quantum mechanics [11. The possibility of realizing such a devices then depends on the ability, for the wave type and wavelength of interest, to fabricate suitable metamaterials and assemble them into the array required by the design.

In this Letter, we propose a new method for designing material parameters having radical effects on waves modeled by Helmholtz-type equations. We refer to this approach as Schrödinger optics, since it is based on the mathematical behavior of quantum mechanical waves, as governed by the Schrödinger equation with a trapping potential. (We stress that Schrödinger optics is not inherently quantum, and applies to any wave phenomena modeled by a Helmholtz equation; the designs most easily realizable using currently available metamaterials are for acoustics and 2D polarized EM.) Different choices of potential in the Schrödinger equation result in different effects on wave propagation, allowing many degrees of freedom. We introduce Schrödinger optics by examining one part of the parameter space, describing designs, both planar and bulk, based on the QM harmonic oscillator in 1D and its degenerate variants. These Schrödinger optics media are striking for the superdimensionality that they exhibit, in which power laws for various physical properties mimic those of a larger dimension than the physical dimension. We focus on two such properties: (i) very high density of resonant frequencies over finite but large frequency bands, a density much larger than that dictated by Weyl's Law [12] for conventional media; and (ii) giant concentration and amplification of waves, resulting from the focussing behavior of the rays in the high frequency limit, controlled by the associated sub-Riemannian geometry [13, 14]. 


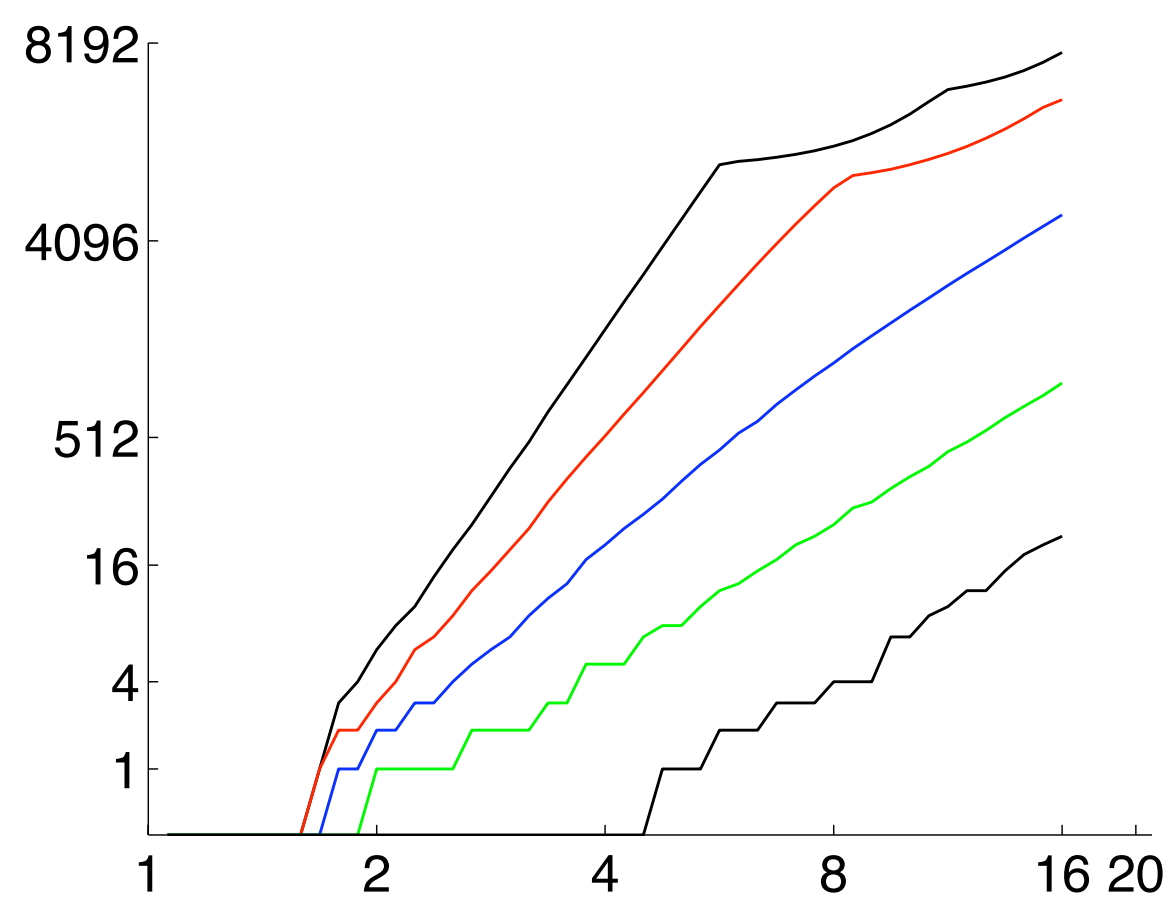

FIG. 1: Superdimensional eigenfrequency density. Log-log graph shows growth of eigenfrequency count functions $N(\omega)$ for five media. The eigenfrequency count functions $N(\omega)$ are shown for approximate SO resonators (4) in the rectangle $R$, with Dirichlet boundary condition, corresponding to parameters $a=0.1$ and $r=4$ (upper black curve), $r=3$ (red curve), $r=2$ (blue curve), and $r=1$ (green curve). The eigenfrequency count function for the homogeneous material corresponding to $r=0$ is shown in the lower black curve. The eigenfrequency count functions display the are superdimensional behavior for $0<\omega<\Omega_{r}=\sqrt{c_{r}} a^{-1}$, with high density of frequencies near $\Omega_{r}$, and the usual 2-dimensional behavior in the high frequency regime $\omega>\Omega_{r}$.

2. Methods. Ideal Schrödinger optics (SO) designs require spatially varying and anisotropic material parameters with infinitely slow wave propagation at some points and in some directions. Recall that the metamaterials required for many transformation optics designs involve parameters at or close to zero and are (for EM) inherently dispersive, or have negative index and are lossy. In contrast, SO designs, since they do not require superluminal phase velocity, are potentially broadband. We analyze both ideal and approximate SO designs, the latter with the potential to be physically realizable (at the price of a quantifiable degradation in performance), but with the characteristic features of ideal SO still present.

2.1. 2D ideal Schrödinger optics metamaterial - We start by considering model SO designs based on quantum oscillators in 1D. Fix an integer $r \geq 1$ and consider a Helmholtz equation 
in $2 \mathrm{D}$ at frequency $\omega>0$ for a wave $u(\mathbf{x})$ on a rectangle $R=\{\mathbf{x}=(x, y):|x| \leq 1,0 \leq y \leq$ $1\}$,

$$
\left(\left(\partial_{x}^{2}+x^{2 r} \partial_{y}^{2}\right)+\omega^{2}\right) u(\mathbf{x})=0
$$

(For $\omega=0$, this equation was first studied by Grushin [15].) We impose the Dirichlet boundary condition (BC) on the boundary of $R, u=0$ for $|x|=1$ or $y=0,1$, representing, e.g., a sound-soft surface in acoustics, but similar results hold for Neumann or mixed BC.

(i) Superdimensionality of eigenfrequency count - For waves propagating in homogeneous material in $d$ dimensions, modeled by $\left(\nabla^{2}+\omega^{2}\right) u=0$, Weyl's Law states that in a $d$ dimensional domain the number of resonant frequencies $\omega_{j}$ grows as $N(\omega):=\#\left\{\omega_{j}: \omega_{j} \leq\right.$ $\omega\} \sim c \cdot \omega^{d}$ [12], where $d$ is the dimension and $c$ is a positive constant; this also holds for general nondegenerate media, for which the mass-density or analogous tensor is nonsingular, so that the resulting PDE is elliptic. There is also a mathematical literature [16] on spectral asymptotics for degenerate-elliptic equations such as (1), but here we derive them directly from eigenvalues and eigenfunctions of quantum harmonic $(r=1)$ and anharmonic $(r \geq 2)$ oscillators.

For solutions to (1) of the form $u(x, y)=\psi_{n}(x) \sin (n \pi y), n=1,2, \ldots$, on $|x| \leq 1$ the $\psi_{n}$ satisfy

$$
L_{n} \psi_{n}:=\left(\frac{d^{2}}{d x^{2}}-\pi^{2} n^{2} x^{2 r}\right) \psi_{n}=-\omega^{2} \psi_{n}, \psi_{n}( \pm 1)=0 .
$$

For now, omit the $\mathrm{BC}$ and consider the same equation on the entire real line $\mathbb{R}$. The operator $L:=d^{2} / d z^{2}-z^{2 r}$ has eigenvalues $\left\{-\lambda_{l}\right\}_{l=1}^{\infty}$ and $L^{2}$-normalized eigenfunctions $\left\{\phi_{l}\right\}_{l=1}^{\infty}$. For $r=1, \lambda_{l}=2 l+1$ and the $\phi_{l}$ are the Hermite functions [12]. For $r \geq 2, \lambda_{l}$ and $\phi_{l}$ are less explicit, but it is known that $\lambda_{l} \sim c_{r} l^{\frac{2 r}{r+1}}$ and $\left|\phi_{l}(z)\right| \leq c_{l, \epsilon} \exp \left(-\frac{|z|^{r+1}}{r+1}(1-\epsilon)\right)$ for any $\epsilon>0$ [17]. Letting

$$
\widetilde{\psi}_{l, n}(x)=(\pi n)^{\frac{1}{2(r+1)}} \phi_{l}\left((\pi n)^{\frac{1}{r+1}} x\right),|x| \leq 1,
$$

$\widetilde{\psi}_{l, n}$ satisfies the ordinary differential equation in 2 with $\omega=\widetilde{\omega}_{l, n}:=$ $(\pi n)^{\frac{1}{r+1}} \lambda_{l}^{\frac{1}{2}} \sim c_{r} n^{\frac{1}{r+1}} l^{\frac{r}{r+1}}$, but with boundary values $\widetilde{\psi}_{l, n}( \pm 1)$ of magnitude $\leq c_{r, \epsilon} \exp \left(-\left(\frac{\pi n}{r+1}-\epsilon\right)\right)$, for any $\epsilon>0$. By standard perturbation theory [18], near $\widetilde{\psi}_{l, n}(x), \widetilde{\omega}_{l, n}$ there exist exact eigenfunctions and eigenfrequencies $\psi_{l, n}(x), \omega_{l, n}$ satisfying (2) and which are exponentially (in $n$ ) close to $\widetilde{\psi}_{l, n}(x), \widetilde{\omega}_{l, n}$; for the purpose of counting resonant frequencies of (1), we may thus work with the $\widetilde{\omega}_{l, n}$ to estimate $N(\omega)$. Ignoring constants, 
$n^{\frac{1}{r+1}} l^{\frac{r}{r+1}} \leq \omega$ if $n \leq \omega^{r+1} / l^{r}$, and the constraint $n \geq 1$ forces $l \leq \omega^{\frac{r+1}{r}}$, so one has

$$
N(\omega) \geq \sum_{l=1}^{\omega^{\frac{r+1}{r}}} \frac{\omega^{r+1}}{l^{r}}=\omega^{r+1} \sum_{l=1}^{\omega^{\frac{r+1}{r}}} \frac{1}{l^{r}}
$$

which is $\sim \omega^{2} \cdot \log \omega$ if $r=1$ and $\sim \omega^{r+1}$ if $r \geq 2$. In comparison with the classical Weyl power law (the growth rate of $N(\omega) \sim \omega^{2}$ for a 2D nondegenerate medium), for $r=1$ this ideal Schrödinger optics medium exhibits a logarithmically greater growth, while for $r \geq 2$, the rate is polynomially greater and in fact is the same as the Weyl Law for $\nabla^{2}$ in dimension $r+1$, see Fig. 1. In summary, the eigenfrequency counting function has the same growth rate as for an $(r+1)$-dimensional resonator.
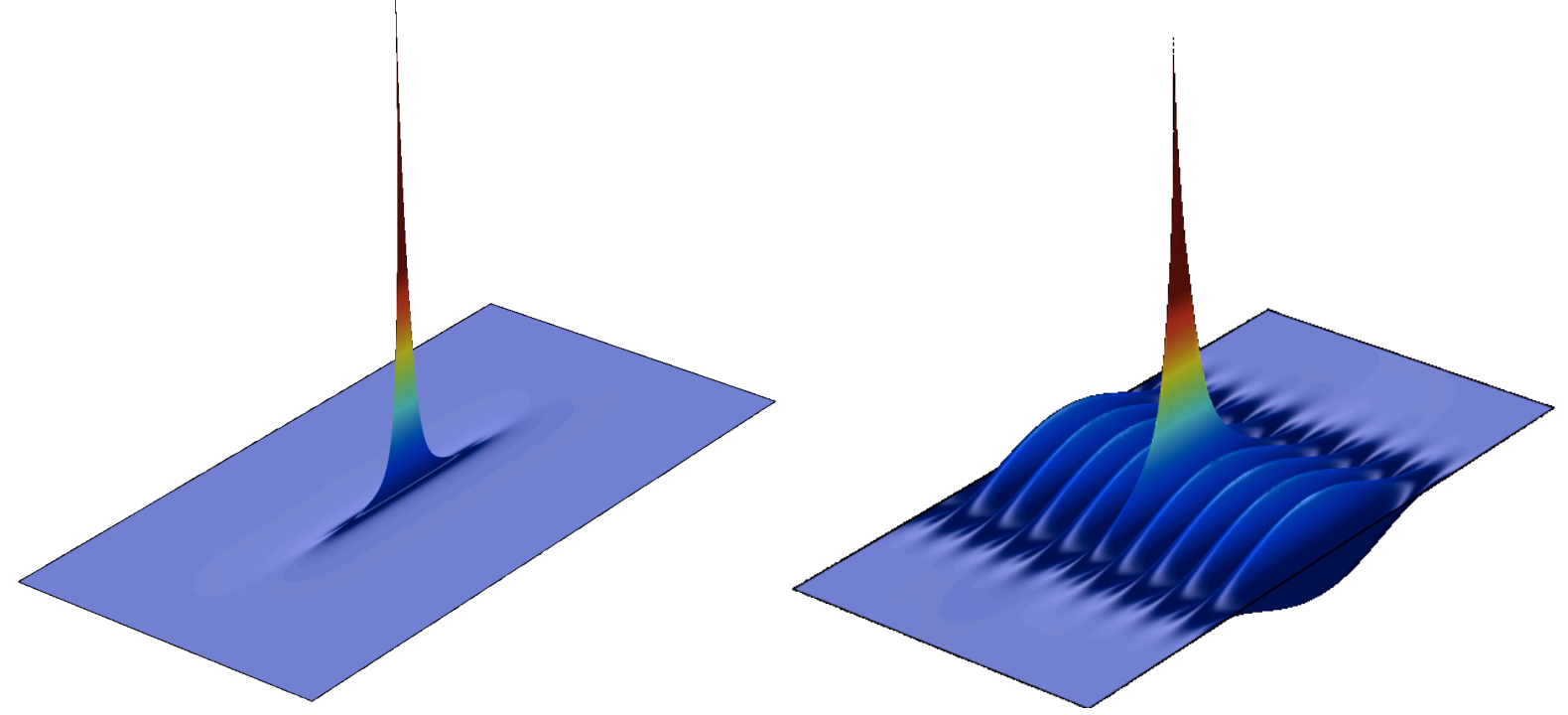

FIG. 2: Superdimensional wave concentration. Green's functions for $r=2$ with pole at point $(0,0)$. The frequency is (left) $\omega=0$ and (right) $\omega=4$. See the [22] for related figures.

(ii) Concentration of waves - SO media also exhibit superdimensionality in the singularity strength of their Green's functions. For $\omega=0$, the Green's function for (1) is known analytically [19] and understood from a geometrical point of view for a more general class of equations [20]. In 2D, the Newtonian potential for a homogeneous, isotropic medium $(r=0), N(\mathbf{x}, \mathbf{y})=\frac{1}{2 \pi} \log |\mathbf{x}-\mathbf{y}|$, is both rotation and translation invariant; fixing the pole at $\mathbf{x}=0$, the singularity is logarithmic and the level curves are circles. In contrast, for $r \geq 1$, the Green's function $G(\mathbf{x}, \mathbf{y})$ for (1) reflects both the inhomogeneity of the medium and the degeneracy along $x=0$. Avoiding mathematical details and extracting from the literature 
only the structure that we need here, write the operator in (1) as $X^{2}+Y^{2}$, where $X, Y$ are the first order differential operators, i.e., vector fields, $X=\frac{\partial}{\partial x}, Y=x^{r} \frac{\partial}{\partial y}$. The flows of these vector fields define a non-Euclidean metric with distance element $d s^{2}=d x^{2}+|x|^{-2 r} d y^{2}$ in the rectangle $R$. This corresponds to the optical length distance, $D(\mathbf{x}, \mathbf{y})$, for the wave propagation, and defines a family of anisotropic "discs", $B(\mathbf{x}, \delta)$, with center $\mathbf{x}$ and "radius" $\delta$. The anisotropic discs are comparable to Euclidean discs away from $x=0$, but those centered along the $y$-axis are comparable to $\delta \times \delta^{r+1}$ rectangles. Analysis shows that in a disc $B(\mathbf{0}, 1)$, of radius one centered at the origin, one can pack $C_{r} \delta^{-(r+1)}$ pairwise disjoint discs of radius $\delta$. The Hausdorff dimension [21] of $R$ endowed with the optical length metric is $r+1$, and the SO material behaves as if it were a higher dimensional space.

We consider the implications of this for the Green's functions. If one denotes by $a(\mathbf{x}, \mathbf{y})$ the Euclidean area of $B(\mathbf{x}, D(\mathbf{x}, \mathbf{y}))$, the Green's function is known to satisfy [23] the estimate

$$
|G(\mathbf{x}, \mathbf{y})| \sim c \cdot\left\{\begin{array}{l}
|\log a(\mathbf{x}, \mathbf{y})|+|\log a(\mathbf{y}, \mathbf{x})|, r=1 \\
\frac{D(\mathbf{x}, \mathbf{y})^{2}}{a(\mathbf{x}, \mathbf{y})}+\frac{D(\mathbf{y}, \mathbf{x})^{2}}{a(\mathbf{y}, \mathbf{x})}, r \geq 2 .
\end{array}\right.
$$

For $r \geq 2$, and $\mathbf{x}$ on the $y$-axis and $\mathbf{y}=\left(y_{1}, y_{2}\right)$, one thus has $|G(\mathbf{x}, \mathbf{y})| \sim\left(\left|y_{1}\right|+\left|y_{2}\right|^{\frac{1}{r+1}}\right)^{-1}$; see Fig. 2. Waves for SO media with point sources on $x=0$ are thus both more singular than the logarithmic blow-up familiar from standard media, and highly concentrated in highly eccentric sets. Similar behavior holds for $\omega \neq 0[22]$.

Next consider the high frequency behavior of the Green's functions as $\omega \rightarrow \infty$. The rays associated to (1) have been calculated [24, 25]. Rays through a given point can only point in directions that are combinations of $X$ and $Y$; thus, passing through $x=0$, motion is only allowed in the $x$ direction. This causes giant focusing of rays: All rays passing through a point $P_{1}$ on that axis $x=0$ focus at $P_{1}$ so that they have a common tangent vector at $P_{1}$ [22]. Moreover, for any two points $P_{1}$ and $P_{2}$ that are on the axis $x=0$, there are infinitely many rays connecting $P_{1}$ and $P_{2}$. Going from the high frequency limit to a finite frequency $\omega$, one sees that a wave produced by a point source at $P_{1}$ focuses strongly at on many points on axis $x=0$ (the focusing depends on the lengths of the connecting rays). This yields the strong concentration and large oscillation of the waves near the axis $x=0$ for large frequencies $\omega$ (c.f. Fig. 2).

Devices with anomalous resonant frequency distributions have been described previously, 
but SO is a fundamentally new approach; e.g., in contrast to fractal antennas [26], SO devices have smooth material parameters and are not self-similar. Designs which strongly concentrate EM waves have also been obtained previously by transformation optics [27].

2.2 Approximate Schrödinger optics - For $0<a<1$,

$$
\left(\partial_{x}^{2}+\left(a^{2 r}+x^{2 r}\right) \partial_{y}^{2}\right) u(\mathbf{x})+\omega^{2} u(\mathbf{x})=0
$$

models a medium approximating the ideal SO medium on the rectangle $R$. The maximum degeneracy occurs at $x=0$ and has moderate contrast in the strip $|x| \leq a$; outside of this strip the approximate medium is close to the ideal. We consider the eigenvalues and eigenfunctions in $R$ with the the Dirichlet $\mathrm{BC} u=0$. Modifying the analysis above, one sees that the spectrum for (2) is shifted by $\pi^{2} n^{2} a^{2 r}$, so that (4) has eigenfrequencies $\omega_{l, n}(a)$ exponentially close to

$$
\widetilde{\omega}_{l, n}(a):=\left(\widetilde{\omega}_{l, n}^{2}+\pi^{2} n^{2} a^{2 r}\right)^{\frac{1}{2}} \sim\left(c_{r}^{2} n^{\frac{2}{r+1}} l^{\frac{2 r}{r+1}}+\pi^{2} a^{2 r} n^{2}\right)^{\frac{1}{2}} .
$$

For $\widetilde{\omega}_{l, n}(a) \leq \omega$, we need both $a^{2 r} n^{2}<\omega^{2}$, so that $1 \leq n \leq a^{-r} \omega$, and $c_{r}^{2} n^{\frac{2}{r+1}} l^{\frac{2 r}{r+1}} \leq \omega^{2}$, so that $n \geq c_{r}^{-1} \omega^{r+1} / l^{r}$. In the low frequency regime, $\omega<\Omega_{r}:=\left(c_{r}\right)^{1 / r} a^{-1}$, the counting function $N(\omega, a):=\#\left\{\omega_{l, n}(a): \omega_{l, n}(a) \leq \omega\right\}$,

$$
N(\omega, a) \sim\left\{\begin{array}{l}
c_{r}^{-1} \omega^{2} \log \omega, r=1, \\
c_{r}^{-1} \omega^{r+1}, r \geq 2,
\end{array}\right.
$$

is superdimensional. For high frequencies, $\omega>\Omega_{r}$,

$$
N(\omega, a) \sim \sum_{l=1}^{c_{r}^{-\frac{1}{r}} a^{\frac{1}{r}} \omega} a^{-1} \omega+\sum_{l=c_{r}^{-\frac{1}{r}} a^{\frac{1}{r}} \omega}^{\infty} c_{r}^{-1} \frac{\omega^{r+1}}{l^{r}} \sim d_{r} a^{\frac{1}{r}-1} \omega^{2}
$$

with $d_{r}=c_{r}^{-1 / r}+\frac{c_{r}^{(r-1) / r}}{r-1}$, is quadratic. For $r \geq 2$ and $\delta>0$, the number of eigenfrequencies in the band $(1-\delta) \Omega_{r} \leq \omega \leq(1+\delta) \Omega_{r}$ is $\sim 2 c_{r}^{-1} \delta \Omega_{r}^{r+1}$, retaining the superdimensionality of the ideal SO medium, while for $\omega \rightarrow \infty$, the quadratic growth rate of $N(\omega, a)$ is as dictated by Weyl's Law in 2D.

As above, the approximate SO medium corresponds to a frequency shift in Helmholtz for the ideal SO medium, $\omega \rightarrow\left(\omega^{2}+a^{2 r}\right)^{\frac{1}{2}}$. When $a$ is small enough, the Green's function is close to the ideal Green's function that has a strong singularity when the source point is at 
the surface $x=0$, and for high frequencies exhibits the ideal medium's strong concentration of rays.

2.3 Implementation - Metamaterial (MM) arrays implementing approximate SO designs at length scale $a<<1$ can be realized using MM atoms composed of rectangles of low speed material on a substrate of high speed material, with increasing vertical fill ratios as $x \rightarrow 0$. See Fig. 3 and the effective medium theory details in [22]. The MM atoms used are nonresonant and thus the standard effective medium approximations used to derive the effective parameters should be valid over a broad band.

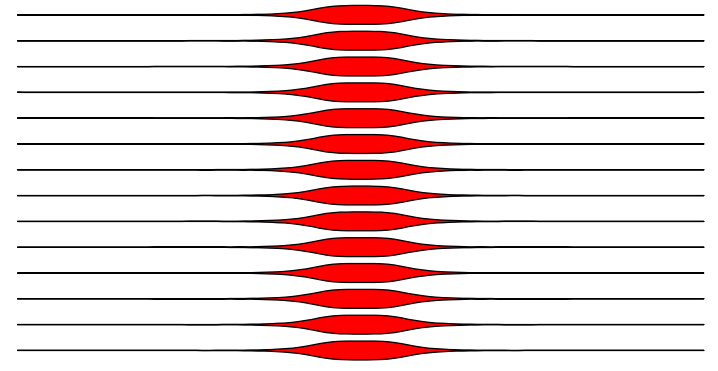

FIG. 3: 2D Schrödinger optics EM metamaterial. Schematic. A lattice of sub-wavelength scale $d \times d$ sized unit atoms, each consisting of an $x$-directed strip of varying width $w(x)$ and high permittivity, embedded in background material with relative permittivity. See [22].

2.4. 3D-Schrödinger optics - An ideal bulk SO material in $Q=\{\mathbf{x}=(x, y, z):|x| \leq$ $1,0 \leq y \leq 1,0 \leq z \leq 1\}$ may be modeled by replacing (1) with $u=0$ on $\partial Q$ and

$$
\left(\partial_{x}^{2}+x^{2 r}\left(\partial_{y}^{2}+\partial_{z}^{2}\right)+\omega^{2}\right) u(\mathbf{x})=0 \text { on } Q
$$

For $\mathbf{n}=\left(n_{1}, n_{2}\right) \in \mathbb{Z}_{+} \times \mathbb{Z}_{+}$, eigenfunctions of the form $u(\mathbf{x})=\psi_{\mathbf{n}}(x) \sin \left(\pi n_{1} y\right) \sin \left(\pi n_{2} z\right)$, $\psi_{\mathbf{n}}$ must satisfy (2) with $n^{2}$ replaced by $\mathbf{n}^{2}:=n_{1}^{2}+n_{2}^{2}$. As above, one constructs approximate spectral data $\widetilde{\psi}_{l, \mathbf{n}}, \widetilde{\omega}_{l, \mathbf{n}}$, admitting exact solution perturbations $\psi_{l, \mathbf{n}}, \omega_{l, \mathbf{n}}$, exponentially nearby (in $|\mathbf{n}|$ ), and to estimate $N(\omega)$, it suffices to work with $\widetilde{\omega}_{l, \mathbf{n}}$. As in the $2 \mathrm{D}$ case, we need both $|\mathbf{n}|^{\frac{1}{r+1}} l^{\frac{r}{r+1}} \leq \omega$, which holds off $|\mathbf{n}| \leq \omega^{r+1} / l^{r}$, and $|\mathbf{n}| \geq 1$, so that $l \leq \omega^{\frac{r+1}{r}}$. Since $\mathbf{n}$ is 2-dimensional,

$$
N(\omega) \geq \sum_{l=1}^{\omega^{(r+1) / r}} \frac{\omega^{2(r+1)}}{l^{2 r}}=c_{r} \omega^{2 r+2}
$$


so that this $3 \mathrm{D}$ ideal SO design exhibits a $(2 r+2)$-dimensional eigenfrequency count. For the least degenerate case, $r=1$, this is already 4-dimensional. As with the 2D case, a more realistic 3D SO design is an approximate one at length scale $a$, with the $x^{2 r}$ in (5) replaced by $a^{2 r}+x^{2 r}$, and this exhibits the superdimensionality in frequency bands about $\Omega=\left(c_{r}\right)^{1 / r} a^{-1}$.

3. Results - The eigenfrequency counting functions for the rectangle $R$ with various media have been computed using Matlab, see Fig. 1. The Green's functions have been evaluated using Comsol and the results are shown in Fig. 2. Documentation of the numerics and more detailed discussion are in [22].

4. Discussion - We have shown that metamaterial arrays that exhibit superdimensional behavior can be designed using the eigenvalue and eigenfunction asymptotics of Schrödinger operators with trapping potentials. The examples presented have axial symmetries and are based on harmonic and degenerate harmonic oscillators in 1D. However, the same principles apply to general nonnegative trapping potentials, in one or higher dimensions, with appropriate spectral asymptotics. Possible applications include components for antennas that have a high density of resonance frequencies in a desired frequency band; materials in which point sources produce fields having extraordinarily strong blow up; and optical materials with giant focussing, that either guide light rays together or separate closely propagating rays.

Acknowledgments: AG is supported by US NSF; YK by UK EPSRC; HK and ML by Academy of Finland; and GU by US NSF, a Walker Family Endowed Professorship at UW and a Clay Senior Award.

[1] A. Greenleaf, M. Lassas and G. Uhlmann, Physiol. Meas. 24, 413 (2003); Math. Res. Lett. 10, 685 (2003).

[2] U. Leonhardt, Sci. 312, 1777 (2006).

[3] J.B. Pendry, D. Schurig, D.R. Smith, Sci. 312, 1780 (2006).

[4] H.-Y. Chen, C.T. Chan, Appl. Phys. Lett. 90, 241105 (2007).

[5] A. Greenleaf, Y. Kurylev, M. Lassas, G. Uhlmann, Phys. Rev. Lett. 99, 183901 (2007).

[6] Y. Lai, et al., Phys. Rev. Lett. 102, 253902 (2009). 
[7] D. Schurig, et al., Sci. 314, 977 (2006).

[8] R. Liu, et al., Sci. 323, 366 (2009).

[9] H.-Y. Chen, C.T. Chan, Appl. Phys. Lett. 90, 241105 (2007); S. Cummer, et al., Phys. Rev. Lett. 100, 024301 (2008); A. Greenleaf, Y. Kurylev, M. Lassas, G. Uhlmann, http://arXiv.org/abs/0801.3279 (2008).

[10] H.-Y. Chen, J. Yang, J. Zi, C. T. Chan, Europhys. Lett. 85, 24004 (2009).

[11] S. Zhang, D. Genov, C. Sun, X. Zhang, Phys. Rev. Lett. 100, 123002 (2008); A. Greenleaf, Y. Kurylev, M. Lassas, G. Uhlmann, Phys. Rev. Lett. 101, 220404 (2008).

[12] M. Taylor, Partial Differential Equations, II, Appl. Math. Sci. 116, Springer, 2006.

[13] R. Strichartz, J. Diff. Geom. 24, 221 (1986).

[14] R. Montgomery, A Tour of Subriemannain Geometries, Their Geodesics and Applications, Amer. Math. Soc., Providence, 2002.

[15] V. Grushin, Mat. Sb. (N.S.) 83, 456 (1970).

[16] A. Menikoff, J. Sjöstrand, Math. Annalen 235, 55 (1978); C. Fefferman, D. H. Phong, Proc. Nat. Acad. Sci. U.S.A. 77, 5622 (1980).

[17] S. Levendorskii, Russ. Math. Surveys 43, 148 (1988); A. Vshivzev, N. Norin, V. Sorokin, Theor. and Math. Phys. 109, 139 (1996).

[18] T. Kato, Perturbation Theory for Linear Operators. Springer-Verlag. Berlin, 1980.

[19] R. Beals, Comm. P.D.E. 24, 369 (1999); R. Beals, P. Greiner, B. Gaveau, J. Func. Anal. 165, 407 (1999).

[20] L. Rothschild, E.M. Stein, Acta Math. 137, 247 (1976).

[21] K. J. Falconer, The geometry of fractal sets. Cambridge Tracts in Math. 85, Cambridge Univ. Pr., 1986.

[22] See Supplemental Material to this paper.

[23] A. Nagel, E.M. Stein, Lectures on Pseudodifferential Operators, Princeton Univ. Pr., 1979.

[24] O. Calin, D.-C. Chang, P. Greiner, Y. Kannai, Contemp. Math. 382, 89 (2005).

[25] D.-C. Chang, Y. Li, Jour. Geom. Anal. 22, 800, (2012).

[26] D. Werner, S. Ganguly, IEEE Ant. Prop. 45, 38 (2003).

[27] U. Leonhardt, T. Tyc, New J. Phys. 10, 115026 (2008). 